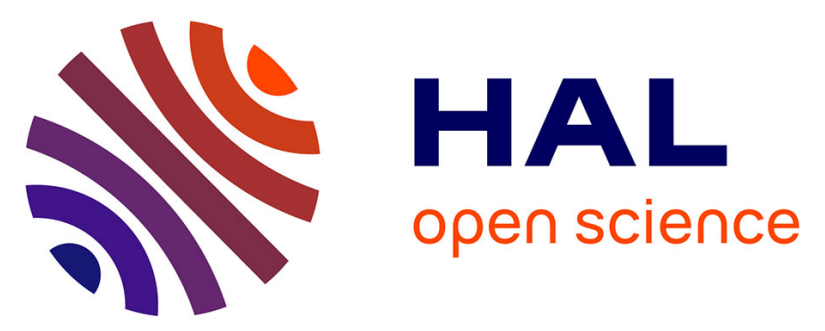

\title{
Risk Factors for Developing Severe Acute Kidney Injury in Adult Patients With Refractory Postcardiotomy Cardiogenic Shock Receiving Venoarterial Extracorporeal Membrane Oxygenation
}

Victoria Lepère, Baptiste Duceau, Guillaume Lebreton, Camille Bombled, Olivier Dujardin, Lucile Boccara, Ahmed Charfeddine, Julien Amour, David Hajage, Adrien Bouglé

\section{- To cite this version:}

Victoria Lepère, Baptiste Duceau, Guillaume Lebreton, Camille Bombled, Olivier Dujardin, et al.. Risk Factors for Developing Severe Acute Kidney Injury in Adult Patients With Refractory Postcardiotomy Cardiogenic Shock Receiving Venoarterial Extracorporeal Membrane Oxygenation. Critical Care Medicine, 2020, 48 (8), pp.e715-e721. 10.1097/CCM.0000000000004433 . hal-02946489

\section{HAL Id: hal-02946489 \\ https://hal.sorbonne-universite.fr/hal-02946489}

Submitted on 23 Sep 2020

HAL is a multi-disciplinary open access archive for the deposit and dissemination of scientific research documents, whether they are published or not. The documents may come from teaching and research institutions in France or abroad, or from public or private research centers.
L'archive ouverte pluridisciplinaire HAL, est destinée au dépôt et à la diffusion de documents scientifiques de niveau recherche, publiés ou non, émanant des établissements d'enseignement et de recherche français ou étrangers, des laboratoires publics ou privés. 


\section{Risk Factors for Developing Severe Acute Kidney Injury in Adult}

\section{Patients with Refractory Post-Cardiotomy Cardiogenic Shock}

\section{Receiving Veno-Arterial Extracorporeal Membrane Oxygenation}

5 Victoria Lepère, $\mathrm{MD}^{1}$, Baptiste Duceau, $\mathrm{MD}^{1}$, Guillaume Lebreton, $\mathrm{MD}, \mathrm{PhD}^{2}$, Camille 6 Bombled, $\mathrm{MD}^{1}$, Olivier Dujardin, $\mathrm{MD}^{1}$, Lucile Boccara, $\mathrm{MSc}^{3}$, Ahmed Charfeddine, $\mathrm{MD}^{1}$, 7 Julien Amour, $\mathrm{MD}, \mathrm{PhD}^{1}$, David Hajage, $\mathrm{MD}, \mathrm{PhD}^{4}$, Adrien Bouglé, $\mathrm{MD}, \mathrm{PhD}^{1}$

$9{ }^{1}$ Sorbonne Université, Assistance Publique - Hôpitaux de Paris (AP-HP), Department of Anesthesiology and Critical Care Medicine, Institute of Cardiology, Pitié-Salpêtrière

11 Hospital, Paris, France

${ }^{2}$ Sorbonne Université, UMR INSERM 1166, IHU ICAN, AP-HP, Department of Cardio-

Vascular and Thoracic Surgery, Institute of Cardiology, Pitié-Salpêtrière Hospital, Paris,

14 France

$15{ }^{3}$ Université Paris Est Créteil, Créteil, France

164 Sorbonne Université, INSERM, Institut Pierre Louis de Santé Publique, Equipe 17 Pharmacoépidémiologie et évaluation des soins, AP-HP, Hôpital Pitié-Salpêtrière, 18 Département Biostatistique Santé Publique Et Information Médicale, Unité de Recherche 19 Clinique PSL-CFX, Centre de Pharmacoépidémiologie (Cephepi), CIC-1421, Paris, France

21 Contact author

22 Correspondence and requests for reprints should be addressed to Adrien Bouglé, M.D., Ph.D., 23 Anesthesiology and Surgical Intensive Care Unit, Cardiology Institute, University Hospital 24 Pitié-Salpêtrière (AP-HP), 47-83 Boulevard de l'Hôpital, 75013 Paris, France. 
1 E-mail: adrien.bougle@aphp.fr

2

\section{Conflict of interest}

4 The authors have not disclosed any potential conflict of interest regarding this study.

5

$6 \quad$ Running title

7 Acute kidney injury in VA-ECMO patients

8

$9 \quad$ Key words

10 Acute Kidney Injury; Extracorporeal Life Support; Cardiac Surgical Procedure;

11 Cardiopulmonary Bypass; Cardiogenic Shock

12

13 Word count

14 Total: 2257 words

15

16

17

18

19

20

21

22

23 


\section{Abstract (326 words)}

2 Objective: Post-cardiotomy cardiogenic shock (PCCS) occurs in 2-6\% of patients undergoing

3 cardiac surgery, and $1 \%$ of cardiac surgery patients will require mechanical circulatory

4 support using Veno-Arterial Extracorporeal Membrane Oxygenation (VA-ECMO). Acute

5 Kidney Injury (AKI) is a frequent complication in this population and negatively impacts the survival. We aimed to determine whether the timing of ECMO implantation influences the renal prognosis of these patients.

Design: Retrospective observational cohort study between January 2013 and December 2016.

9 Setting: An 18-bed surgical ICU in a university hospital.

10 Patients: 4796 consecutive adult patients that underwent cardiac surgery were included in the 11 study and $347(7.2 \%)$ were assisted with VA-ECMO for refractory PCCS. The patients that died during the first 48 hours after VA-ECMO implantation were excluded. The complete-

13 case analysis included 257 patients.

14 Interventions: None.

15 Measurements: The primary outcome was the occurrence, within ten days following the VA16 ECMO implantation, of a stage 3 AKI defined by the Kidney Disease: Improving Global 17 Outcomes (KDIGO) group.

Main results: One hundred sixty-nine patients $(65.7 \%)$ presented with a KDIGO stage 3 AKI; 14 patients $(5.4 \%)$ died before the end of the follow-up period, without developing the primary outcome. $92 \%$ of patients with KDIGO 3 AKI received RRT, for a median duration

21 of 7 [3, 16] days. Late implantation of VA-ECMO was independently associated with an increased risk of KDIGO stage 3 AKI (Odds Ratio 2.81 [95\% CI 1.31, 6.07], $P=0.008$ ). The other factors associated with KDIGO stage 3 AKI were pre-operative left ventricular ejection 24 fraction, OR 1.03 [95\% CI 1.01, 1.05], $P=0.007$; intraoperative plasma transfusion OR 1.13 [95\% CI 1.02, 1.26], $P=0.022$, increased bilirubinemia level OR 1.013 [95\% CI 1.001, 
1 1.026], $P=0.032$, and increased creatinine levels OR 1.012 [95\% CI 1.006, 1.018], $P<0.001$

2 on the day of implantation.

3 Conclusions: Significant kidney dysfunction is particularly frequent in patients with 4 refractory PCCS assisted with VA-ECMO. Early implantation of ECMO may help prevent 5 acute kidney injury.

6

7 Trial Registration: AKI ECMO Trial Registration: ClinicalTrials.gov Identifier $8 \quad$ NCT04082312 


\section{ABBREVIATIONS}

2 ACT, Activated whole blood Clotting Time

3 AKI, Acute Kidney Injury

4 ASA, American Society of Anesthesiologists

5 BMI, Body Mass Index

6 CI, Confidence Intervals

7 COPD, Chronic Obstructive Pulmonary Disease

8 DO2, Dissolved O2

9 ECMO, ExtraCorporeal Membrane Oxygenation

10 ELSO, Extracorporeal Life Support Organization

11 KDIGO, Kidney Disease: Improving Global Outcomes

12 LVEF, Left Ventricular Ejection Fraction

13 OR, Odds Ratio

14 PCCS, Post-Cardiotomy Cardiogenic Shock

15 pCO2, CO2 Partial Pressure

16 pO2, O2 Partial Pressure

17 RRT, Renal Replacement Therapy

18 SAPS 2, simplified acute physiology score 2

19 SOFA, Sepsis-related Organ Failure Assessment

20 VA-ECMO, Veno-Arterial ExtraCorporeal Membrane Oxygenation 


\section{INTRODUCTION}

2 Cardiogenic shock occurs in $2-6 \%$ of patients undergoing cardiac surgery (1). Approximately

$31 \%$ of patients will require mechanical support using Veno-Arterial ExtraCorporeal

4 Membrane Oxygenation (VA-ECMO) owing to refractory post-cardiotomy cardiogenic shock

5 (PCCS) (2). Post-cardiotomy VA-ECMO is associated with a high morbidity that includes

6 bleeding, ischemic events, and organ failure including acute kidney injury (AKI) (3). Indeed,

7 AKI is one of the most frequent complications in patients supported by ECMO, with an

8 incidence reaching up to $65 \%$ (4). Renal replacement therapy (RRT) is required in

9 approximately $65 \%$ of patients (3), and is associated with increased mortality (5).

10 In the case of PCCS, two perioperative mechanisms may play a role in the development of

11 AKI. First, low cardiac output associated with left ventricular failure leads to a decline in

12 renal blood flow and glomerular filtration. Second, right ventricular failure may also impair

13 glomerular filtration due to renal congestion. Both renal congestion and low cardiac output 14 are treated by VA-ECMO which helps increase flow rate and arterial pressure while 15 decreasing central venous pressure.

16 Through these protective mechanisms the timing of VA-ECMO implantation may influence 17 the prognosis of patients with refractory PCCS. Our hypothesis was that early implantation of 18 VA-ECMO may decrease the incidence of AKI in patients with PCCS. The aim of our study 19 was to describe the incidence of acute kidney injury in adult patients with refractory PCCS 20 supported by VA-ECMO and identify the risk factors for developing AKI. 


\section{METHODS}

2 This study was approved by the ethics committee Ile de France 5 (reference B-7-15). Due to

3 the retrospective design of the study, and in accordance with the decision of the ethics

4 committee and French regulation and non-interventional studies, signed informed consent was

5 waived. The study was conducted in accordance with the Declaration of Helsinki principles.

Design and setting

8 This observational single center retrospective study was conducted in the Surgical Intensive

9 Care Unit of the Cardiology Institute of La Pitié-Salpêtrière University Hospital (Paris,

France). All patients who underwent cardiac surgery admitted in our unit and assisted by VAECMO between January 2013 and December 2016 were screened. The exclusion criteria were

i) chronic hemodialysis, ii) death occurring within the first 48 hours of VA-ECMO implantation and iii) multiple VA-ECMO episodes during the same hospitalization. Patients with VA-ECMO were identified through billing record in the electronic medical records system. All patient data was anonymized.

Main goals and Outcomes

Our primary objective was to describe the incidence of acute kidney injury in adult patients with refractory PCCS supported by VA-ECMO and identify the risk factors for developing AKI. The primary outcome was the occurrence of a stage 3 AKI defined by the Kidney Disease: Improving Global Outcomes (KDIGO) group (Supplemental Appendix) (6) within ten days of VA ECMO implantation. Stage 3 AKI was selected due to its potential for requiring RRT. During this ten days period (D1 to D10), AKI and death behave as competitive events (D1 represents the day following the implantation of ECMO). The primary outcome group was divided as follows (i) patients with stage 3 AKI within 10 days of VA 
1 ECMO implantation (group 1); (ii) patients without stage 3 AKI 10 days after VA ECMO

2 implantation (group 2, baseline category); (iii) patients that died between D2 and D10 of VA-

3 ECMO support, without developing KDIGO stage 3 AKI (group 3). Death occurring before

4 D2 of VA-ECMO implantation was an exclusion criterion.

5 Our second goal was to determine the short-term mortality risk factors in this population. The

6 secondary outcome was defined as 30-day mortality after VA-ECMO implantation. Long-

7 term outcomes including the 6-month mortality rate, and the 1-month and 6-month renal 8 prognosis were also assessed.

10 Statistical analysis

11 Results are expressed as number of patients (\%), median (interquartile range 25-75) or mean

12 ( \pm standard deviation). Shapiro-Wilk test was used to assess the normality assumption for 13 continuous variables. The groups were compared using Kruskal-Wallis, Pearson Chi-square 14 and Fisher tests. Univariable analyses and multivariable regression models were used to identify the risk factors for developing the primary and the secondary outcomes.

Risk factors analyzed included demographic factors (SAPS 2, SOFA, Pre-operative LVEF, chronic kidney failure, creatinine clearance, type of surgery), hemodynamic factors during intraoperative period (cardiopulmonary bypass time, transfusion, catecholamines), factors associated with VA-ECMO implantation (primary graft dysfunction, lactate, intraoperative or postoperative implantation, catecholamines), and biological factors at D1 of VA-ECMO

21 support (creatinine, blood protein level, bilirubinemia, bicarbonates, lactate, urine output).

22 A multivariate logistic regression was used to model the occurrence of stage 3 AKI. This 23 model was chosen to take into account the competing risk of death without developing a stage 243 AKI. The three levels consisted of patients with KDIGO stage 3 AKI (group 1), patients without KDIGO stage $3 \mathrm{AKI}$ and alive at D10 (group 2 [baseline category]), and patients that 
1 died before D10 without developing KDIGO stage 3 AKI (group 3). Candidate risk factors

2 associated $(\mathrm{p}<0.05)$ with this outcome in the univariable analysis were introduced into the

3 multivariate model, and the final model was selected using backward stepwise selection

4 method.

5 Risk factors for the 30-day mortality were demographic factors (age, BMI, SAPS 2, SOFA,

6 LVEF, diabetes, dyslipidemia, hypertension, peripheral artery disease), intraoperative factors

7 (transfusion), variables at VA-ECMO implantation (biventricular failure, primary graft

8 dysfunction, lactate, inhaled nitric oxide, AKI at implantation, urine output), and type of

9 surgery (heart transplantation, valve surgery, coronary bypass surgery, combined surgery).

10 Regarding the secondary outcome, the final logistic regression model was obtained using the

11 same selection method as used for the analysis of the primary outcome.

12 Discrimination abilities was quantified using the Polytomous Discrimination Index (PD13 Index) (7) for the final multinomial model of the occurrence of stage $3 \mathrm{AKI}$, and the

14 Concordance Index (C-Index) (7) for the binary logistic model of 30-day mortality. Internal

15 validation was performed with a bootstrap procedure using 500 repetitions (8), to quantify the

16 optimism that may be expected when the multivariable model is applied to new, but similar,

17 patients. Both apparent and optimism-corrected discrimination indexes were reported

18 Odds Ratio (OR) with their $95 \%$ confidence intervals (CI) was calculated. The complete-case method was used for the multivariable analyses. A sensitivity analysis using multiple imputations replaces missing values when appropriate was also performed (Supplemental

21 Table 2). All tests were 2-sided and a $P$-value $<0.05$ was considered for statistical 22 significance. Statistical analyses were performed using R software (version 3.4.1, licenses GNU GPL, The R foundation for statistical computing, Vienna, Austria). 


\section{RESULTS}

\section{Study population}

3 Between January 2013 and December 2016, 4796 patients underwent cardiac surgery with 4 cardiopulmonary bypass. Among them, 347 (7.2\%) experienced refractory PCCS and 5 required VA-ECMO assistance (peripheral cannulation 94.8\%) (Figure 1, flowchart).

7257 patients were included in the final analysis. Demographic and outcome variables did not 8 significantly differ between the patients included and excluded from the analysis. One 9 hundred sixty-nine (65.7\%) patients developed KDIGO stage 3 AKI within 10 days of VAECMO support. Demographic and clinical characteristics of the population are described in

11 Table 1. The clinical management of VA-ECMO patients is described in the Supplementary 12 Appendix.

\section{$14 \quad$ VA-ECMO implantation}

15 Table 2 shows data regarding perioperative management of the patients. The VA-ECMO was 16 implanted intraoperatively in 159 patients (61.9\%) and postoperatively in 98 patients (38.1\%). 17 A left ventricular venting was necessary in 105 patients (40.9\%), most commonly an intra18 aortic balloon pump per institutional protocol (No.=92, 35.8\%). The causes of VA-ECMO 19 implantation were left ventricular failure (No.=76, 29.6\%), right ventricular failure (No.=45, $2017.5 \%)$, biventricular failure $($ No. $=88,34.2 \%)$, primary graft failure $($ No. $=30,11.7 \%)$ or 21 cardiac arrest $($ No. $=15,5.8 \%)$. 
1 During the follow-up period of ten days after VA-ECMO support, 169 patients $(65.7 \%)$

2 developed a KDIGO stage 3 AKI; 74 patients (28.8 \%) did not develop a KDIGO stage 3 AKI

3 and were still alive at D10; 14 patients $(5.4 \%)$ died before the end of the follow-up period,

4 without developing the primary outcome. Renal replacement therapy was initiated in 156

5 patients $(60.7 \%)$ from the overall cohort. $92 \%$ of patients with KDIGO stage 3 AKI received

6 RRT for indications including ionic disorders $($ No. $=79,46.7 \%)$, anuria $($ No. $=61,36.1 \%)$ and

7 fluid overload (No.=15, 8.9\%). The median duration of RRT was 7 [IQR 3, 16] days.

8 Results from the univariate analysis assessing risk factors for developing KDIGO stage 3 AKI

9 are presented in Table 2. SOFA, SAPS2, and urine output at Day 1 were excluded from the 10 analysis due to a lack of data ( $>15 \%$ of data points missing). Figure 2 summarizes the results

11 of the multivariate logistic regression. Factors independently associated with the development

12 of KDIGO stage 3 AKI were bilirubinemia and creatinine levels on the day of ECMO 13 implantation, the timing of ECMO implantation, pre-operative LVEF and intraoperative fresh 14 frozen plasma transfusion. Complete multivariate results including group 3 patients is 15 available in the Supplemental Table 1. The Polytomous Discrimination Index (PD-Index) for 16 the final multivariate model of the occurrence of stage 3 AKI was 0.624. After internal 17 validation, the optimism-corrected discrimination index was 0.294.

\section{Risk factors for 30-day mortality}

The 30 day mortality rate was $48.2 \%$ (124/257). The timing of VA-ECMO implantation did

21 not reduce the mortality at D30 in the univariate analysis, with an intraoperative mortality rate of $44.7 \%($ No.=71/159) vs. $54.1 \%($ No.=53/98) for postoperative implantation OR 0.69 [0.40, 1.17] $P=0.16$. The 6 month mortality rate was $75.9 \%(n=154 / 203)$. Intraoperative

24 implantation of VA-ECMO was associated with a lower mortality at 6 months, 69.8\% (No. $25=88 / 126,20.8 \%$ missing status) compared to $85.7 \%$ ( $n=66 / 77,21.4 \%$ missing status) for 
1 patients with postoperative implantation, $\mathrm{OR}=0.39[0.17,0.85], P=0.011$. The factors

2 associated with 30-day mortality after VA-ECMO implantation are presented in the Table 3.

3 The Concordance Index (C-Index) for the binary logistic model of 30-day mortality was

4 0.721. After internal validation, the optimism-corrected discrimination index was 0.482 .

5 Renal prognosis at one month (Supplemental Table 3) was good, with all but three patients

6 recovered from their stage 3 AKI at 6 months.

7

8

9

10

11

12

13

14

15

16

17 


\section{DISCUSSION}

2 To our knowledge, this study is the first to evaluate the risk factors for developing AKI in

3 adult patients with refractory PCCS. In this population, intra-operative implantation of VA-

4 ECMO appeared to be a protective factor for developing a stage 3 AKI.

5 The incidence of KDIGO stage 3 AKI in our cohort was $65.7 \%$. This is higher than prior reported values from postoperative cardiac surgical patients without VA-ECMO assistance (9), but consistent with the results from Yan et al., who found an incidence of severe AKI of $48 \%$ at 48 hours after VA-ECMO implantation (10). The use of renal replacement therapy was higher than previously described, with initiation of RRT in $60.7 \%$ of patients in the overall cohort (11). Typically, patients needing VA-ECMO support have a higher comorbidity index and lower pre-operative LVEF. These patients are thus at higher risk for postoperative complications, including AKI. Additionally, the relatively high incidence of refractory PCCS in our cohort reflects the underlying health status of the population of an expert university hospital, with mean EuroSCORE II (12) predicting the intraoperative mortality of almost $20 \%$.

The results of this study magnify the importance of the timing around implantation of VAECMO support. We found that intraoperative implantation of VA-ECMO had a protective effect on the development of KDIGO stage 3 AKI. Intraoperative implantation of VA-ECMO limits the duration of the low cardiac output associated with PCCS and thus helps prevent subsequent organ dysfunction. Hence, biological marker of organ dysfunction as

21 hyperbilirubinemia was independent risk factor of KDIGO stage $3 \mathrm{AKI}$ in our cohort.

Due to the design of our study, we were not able to assess the effect of developing an AKI 3 between D2-D10 on the 30 day mortality rate. The overlap between the exposition period to 24 AKI and the mortality risk prevented modeling of the relationship between these two variables. Nonetheless this association has been extensively documented in past studies (13- 
1 15). Given the high incidence of AKI, with $89 \%$ of patients developing at least KDIGO stage

21 AKI, preventative strategies may have a major impact on patient outcomes. The other 30-

3 day mortality risk factors highlighted by our study are commonly described in both a post-

4 cardiotomy population (16) and in VA-ECMO population [(15);(17)], including age, BMI, the

5 presence of chronic arteriopathy and biventricular failure. The supplemental figure (Diagram

6 Acyclic Graph) summarizes the results and the hypotheses stemming from this study and

7 provides additional context with respect to cardiac surgery-associated AKI.

8 Our study has several limitations. First, because of its retrospective and single center design,

9 unidentified confounding factors may have biased the multivariable analyses. Nonetheless,

10 the risk factors we identified are consistent with the ones previously described, providing us

11 with some external validity. Second, if missing data did not allow a full-set analysis, we used

12 a sensitivity analysis using multiple imputations to replaces missing values. Third, the lack of

13 long term follow-up, inherent to the retrospective design, prevented us from making

14 conclusions regarding the effect of long-term mortality or renal outcome. Fourth, there was no

15 detailed protocol regarding cardiopulmonary bypass weaning, decision for VA-ECMO

16 implantation in case of refractory PCCS, or RRT initiation in the intensive care unit.

17 However, our practices are relatively standardized, and it is unlikely that a patient with

18 refractory PCCS during the perioperative period would not have been assisted by VA-ECMO. 


\section{CONCLUSIONS}

2 In conclusion, this retrospective study is the first to emphasize the role of intraoperative VA-

3 ECMO support in case of refractory PCCS, which could possibly decrease the incidence of

4 KDIGO stage 3 AKI. AKI is the easier read-out of low cardiac output for intensivist, and

5 other organ dysfunction might be avoided with early VA-ECMO support. Only a prospective

6 study with randomization could definitely clarify the issue of timing in VA-ECMO support

7 during PCCS.

8

9 


\section{ACKNOWLEDGMENTS}

2 None.

3

4 


\section{REFERENCES}

1. Muehrcke DD, McCarthy PM, et al: Extracorporeal membrane oxygenation for postcardiotomy cardiogenic shock. Ann Thorac Surg. 1996 Feb;61(2):684-91.

2. Smith C, Bellomo R, Raman JS, et a:. An extracorporeal membrane oxygenationbased approach to cardiogenic shock in an older population. Ann Thorac Surg. 2001 May;71(5):1421-7.

3. Rastan AJ, Dege A, Mohr M, et al: Early and late outcomes of 517 consecutive adult patients treated with extracorporeal membrane oxygenation for refractory postcardiotomy cardiogenic shock. J Thorac Cardiovasc Surg. 2010 Feb;139(2):302-11, 311.e1.

4. Haneya A, Diez C, Philipp A, et al: Impact of Acute Kidney Injury on Outcome in Patients With Severe Acute Respiratory Failure Receiving Extracorporeal Membrane Oxygenation. Crit Care Med. 2015 Sep;43(9):1898-906.

5. Ko W-J, Lin C-Y, Chen RJ, et al: Extracorporeal membrane oxygenation support for adult postcardiotomy cardiogenic shock. Ann Thorac Surg. 2002 Feb;73(2):538-45.

6. Kellum JA, Lameire N, Aspelin P, et al: Kidney disease: Improving global outcomes (KDIGO) acute kidney injury work group. KDIGO clinical practice guideline for acute kidney injury. Kidney Int Suppl. 2012 Mar 1;2(1):1-138.

7. Van Calster B, Vergouwe Y, Looman CWN, et al: Assessing the discriminative ability of risk models for more than two outcome categories. Eur J Epidemiol. 2012 Oct;27(10):76170.

8. Steyerberg EW, Eijkemans MJ, Harrell FE, et al: Prognostic modelling with logistic regression analysis: a comparison of selection and estimation methods in small data sets. Stat Med. 2000 Apr 30;19(8):1059-79.

9. Wang Y, Bellomo R: Cardiac surgery-associated acute kidney injury: risk factors, pathophysiology and treatment. Nat Rev Nephrol. 2017 Nov;13(11):697-711.

10. Yan X, Jia S, Meng X, et al: Acute kidney injury in adult postcardiotomy patients with extracorporeal membrane oxygenation: evaluation of the RIFLE classification and the Acute Kidney Injury Network criteria. Eur J Cardiothorac Surg. 2010 Feb 1;37(2):334-8.

11. Elella RA, Habib E, Mokrusova P, et al: Incidence and Outcome of Acute Kidney Injury by the pRIFLE Criteria for Children Receiving Extracorporeal Membrane Oxygenation after Heart Surgery. Ann Saudi Med. 2017 Jun;37(3):201-6.

12. Nashef SAM, Roques F, Sharples LD, et al: EuroSCORE II. Eur J Cardio-Thorac Surg. 2012 Apr;41(4):734-44; discussion 744-745.

13. Hobson C, Ozrazgat-Baslanti T, Kuxhausen A, et al: Cost and Mortality Associated With Postoperative Acute Kidney Injury. Ann Surg. 2015 Jun;261(6):1207-14.

14. Mao H, Katz N, Ariyanon W, et al: Cardiac surgery-associated acute kidney injury. Cardiorenal Med. 2013 Oct;3(3):178-99.

15. Shah SA, Shankar V, Churchwell KB, et al: Clinical outcomes of 84 children with congenital heart disease managed with extracorporeal membrane oxygenation after cardiac surgery. ASAIO 1992. 2005 Oct;51(5):504-7.

16. Weisel RD, Nussmeier N, Newman MF, et al: Predictors of contemporary coronary artery bypass grafting outcomes. J Thorac Cardiovasc Surg. 2014 Dec;148(6):2720-2726.e12.

17. Papadopoulos N, Marinos S, El-Sayed, et al: Risk factors associated with adverse outcome following extracorporeal life support: analysis from 360 consecutive patients. Perfusion. 2015 May;30(4):284-90. 


\section{FIGURE LEGENDS}

2 Figure 1. Flowchart

3 This flowchart shows the distribution of the patients among the three groups according to the

4 primary outcome. AKI was defined by the KDIGO classification (6). D10 refers to the tenth

5 day after VA-ECMO implantation. Death before D2 was an exclusion criterion

6 AKI, Acute Kidney Injury; ICU, Intensive Care Unit.

7

8

9 Figure 2. Adjusted odds ratios of KDIGO stage 3 Acute Kidney Injury.

10 Forrest plot of risk-adjusted odd ratios of stage 3 Acute Kidney Injury. Bilirubinemia and

11 creatininemia refer to the worst values on the day of VA-ECMO implantation.

12 LVEF, Left Ventricular Ejection Fraction

13

14

15

16

17

18

19

20

21 


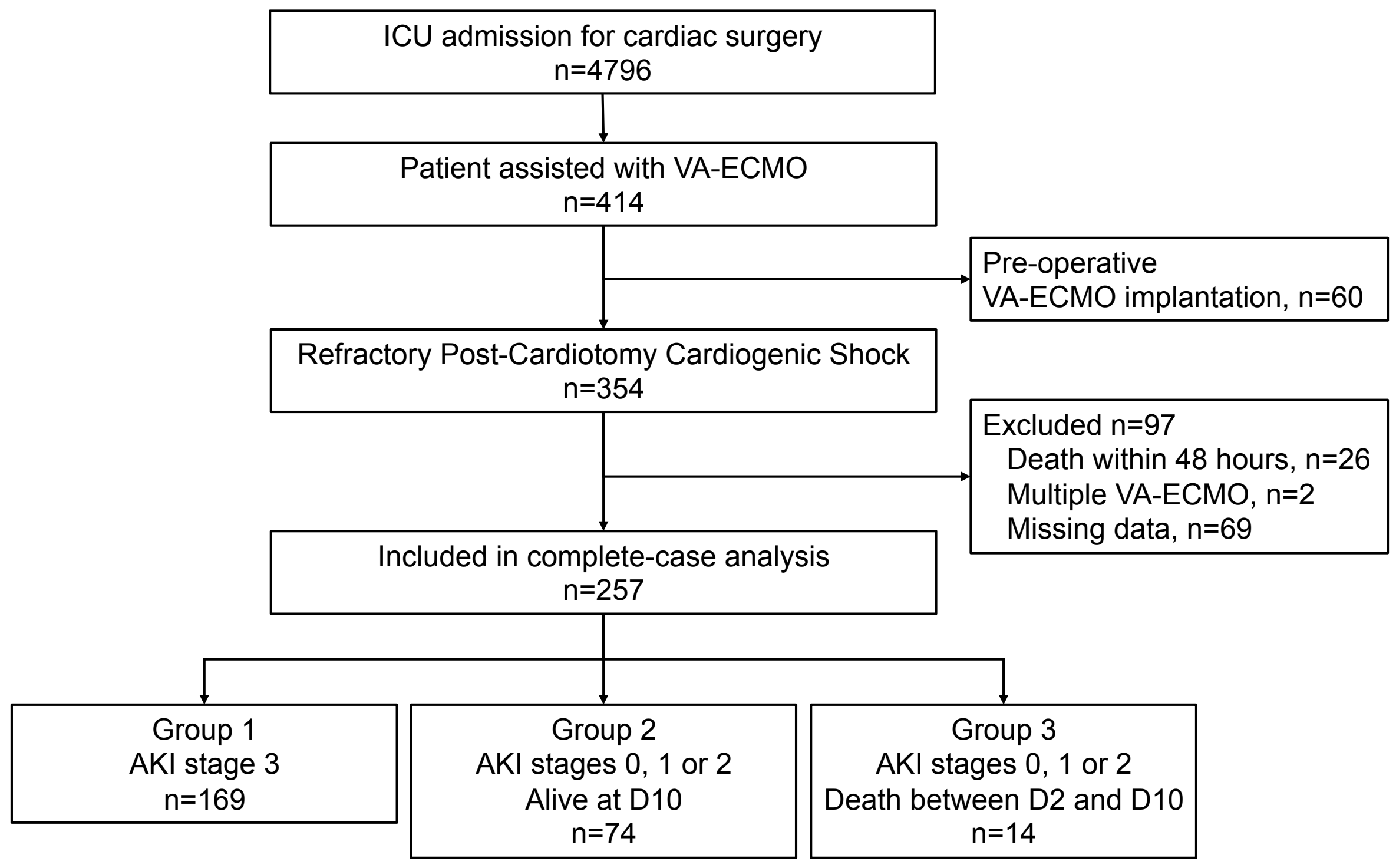


LVEF (per \% increase)

Creatininemia (per $\mu \mathrm{mol} / \mathrm{L}$ increase)

Bilirubinemia (per $\mu \mathrm{mol} / \mathrm{L}$ increase)

Postoperative implantation of VA-ECMO

Intraoperative fresh frozen plasma (per unit $=200 \mathrm{~mL}$ )

\section{Odds Ratio [95\% Cl] P-value}

$\begin{array}{cc}1.03[1.01,1.05] & 0.007 \\ 1.012[1.006,1.018] & <0.001 \\ 1.013[1.001,1.026] & 0.032 \\ 2.81[1.31,6.07] & 0.008 \\ 1.13[1.02,1.26] & 0.022\end{array}$

0.022

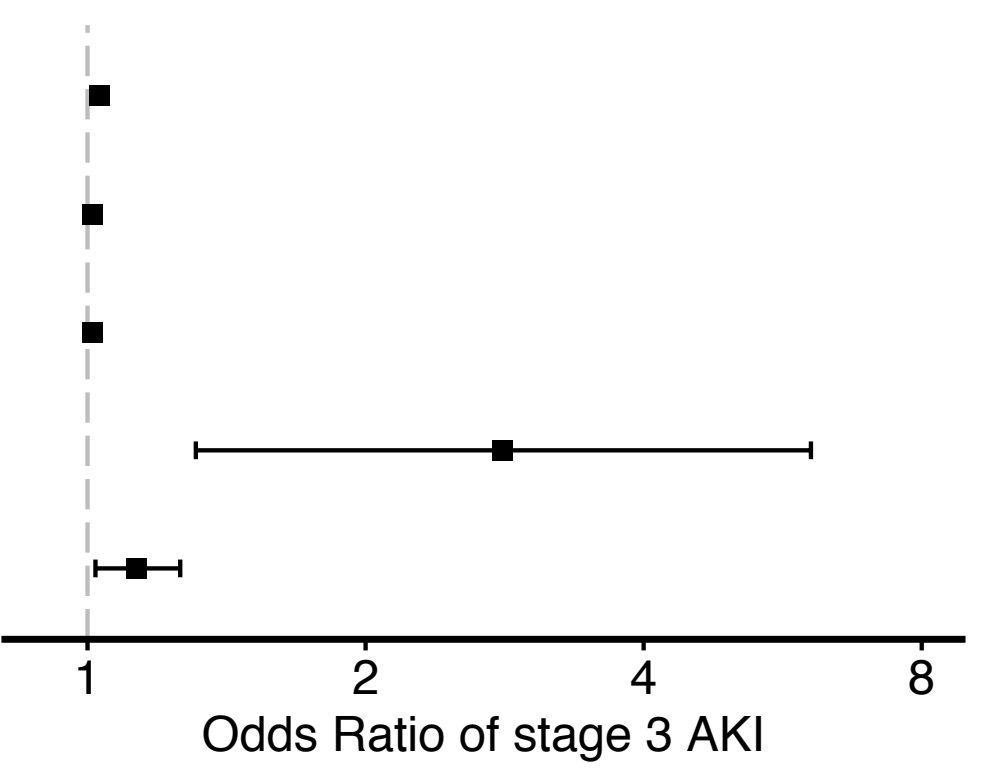


Supplemental Table 1: Multivariable analysis of risk factors for AKI in patients going under cardiac surgery supported by VA-ECMO

\section{Factors}

Outcome Odds Ratio [95\%

CI]

p-value

Global p-

value

\begin{tabular}{|c|c|c|c|c|}
\hline \multirow{2}{*}{ Preoperative LVEF, per \% increase } & Group 1 & $1.029[1.008,1.050]$ & 0.007 & \multirow{2}{*}{0.021} \\
\hline & Group 3 & $1.025[0.984,1.068]$ & 0.241 & \\
\hline \multirow{2}{*}{ Post-operative vs per-operative VA-ECMO } & Group 1 & $2.819[1.308,6.073]$ & 0.008 & \multirow{2}{*}{0.005} \\
\hline & Group 3 & $0.409[0.044,3.795]$ & 0.431 & \\
\hline \multirow{2}{*}{ Bilirubinemia ${ }^{\mathrm{a}}$, per $\mu \mathrm{mol} / \mathrm{L}$ increase } & Group 1 & $1.013[1.001,1.026]$ & 0.032 & \multirow[b]{2}{*}{0.006} \\
\hline & Group 3 & $0.971[0.928,1.016]$ & 0.209 & \\
\hline \multirow[b]{2}{*}{ Creatininemia ${ }^{\mathrm{b}}$, per $\mu \mathrm{mol} / \mathrm{L}$ increase } & Group 1 & $1.012[1.006,1.018]$ & 0.0001 & \multirow[b]{2}{*}{0.0001} \\
\hline & Groun 3 & $1009[0.998,1.021]$ & 0.099 & \\
\hline \multirow{3}{*}{ Per-operative fresh frozen plasma transfusion } & Group 1 & $1.133[1.18,1.262]$ & 0.022 & \multirow{3}{*}{0.019} \\
\hline & & & & \\
\hline & Group 3 & $1.229[1.032,1.464]$ & 0.021 & \\
\hline
\end{tabular}

Multinomial logistic regression modeling renal status within 10 days of VA-ECMO implantation for refractory pstcardiotomy cardiogenic shock. Group 1 : patients that presented the primary outcome of KDIGO stage 3 AKI; Group 2 [baseline category] : patients that did not experience the primary outcome and that were still at risk of presenting the primary outcome at D10, e.g. were still alive at D10; Group 3 : patients that died between D2 and D10 of VA-ECMO support, without developing KDIGO stage 3 AKI.

a at the time of VA-ECMO implantation

$\mathrm{b}$ at day one after VA-ECMO implantation

LVEF, Left Ventricular Ejection Fraction; VA-ECMO, Veno-Arterial ExtraCorporeal Membrane Oxygenation 
Table 2: Univariable analysis of potential risk factors for KDIGO stage 3 AKI

\begin{tabular}{|c|c|c|c|c|}
\hline \multirow[b]{2}{*}{ Factors } & \multirow{2}{*}{$\begin{array}{l}\text { Patients with } \\
\text { stage } 3 \text { AKI } \\
\text { (No.=169) }\end{array}$} & \multicolumn{2}{|c|}{ Patients without stage 3 AKI } & \multirow[b]{2}{*}{$P$-value } \\
\hline & & $\begin{array}{c}\text { alive } \\
\text { at D10 } \\
\text { (No.=74) }\end{array}$ & $\begin{array}{c}\text { deceased } \\
\text { before D10 } \\
(\text { No.=14) }\end{array}$ & \\
\hline \multicolumn{5}{|c|}{ Demographic characteristics and comorbidities } \\
\hline SAPS 2 & $61.1 \pm 18.3$ & $53.7 \pm 16.7$ & $56.4 \pm 19.2$ & 0.043 \\
\hline SOFA & $16.8 \pm 3.2$ & $11.7 \pm 2.9$ & $12.6 \pm 3.9$ & $<0.0001$ \\
\hline Preoperative LVEF (\%) & $37.3 \pm 16.9$ & $30.6 \pm 16.7$ & $36.9 \pm 16.6$ & 0.011 \\
\hline Chronic kidney failure $^{\mathrm{a}}$ & $46(27.2)$ & $21(28.4)$ & $2(14.2)$ & 0.61 \\
\hline Creatinine clearance, $\mathrm{ml} / \mathrm{min}$ & $68[46,89]$ & $73[55,97]$ & & 0.15 \\
\hline Type of surgery & & & & 0.0503 \\
\hline Heart transplant & $29(17.2)$ & $28(37.8)$ & $2(14.3)$ & \\
\hline Valvular surgery & $51(30.2)$ & $15(20.3)$ & $4(28.6)$ & \\
\hline Coronary bypass & $36(21.3)$ & $11(14.8)$ & $2(19.0)$ & \\
\hline Combined surgery & $17(10.0)$ & $4(5.4)$ & $1(7.1)$ & \\
\hline Others & $36(21.3)$ & $16(21.6)$ & $5(35.7)$ & \\
\hline \multicolumn{5}{|l|}{ Intraoperative period } \\
\hline Cardiopulmonary Bypass Time, min & $123[87,181]$ & $123[89,166]$ & $123[78,158]$ & 0.85 \\
\hline Red Blood Cell transfusion, number & $2[0,5]$ & $2[0,4]$ & $3[2,6.5]$ & 0.37 \\
\hline Platelet transfusion, number & $1[0,1]$ & $1[0,1]$ & $1[0.25,1.75]$ & 0.68 \\
\hline Plasma transfusion, number & $3[0,6]$ & $3.5[0,5]$ & $6.5[3,8.75]$ & 0.047 \\
\hline Cell salvage autologous transfusion, $\mathrm{mL}$ & $450[230,682]$ & $423[240,533]$ & $717[315,840]$ & 0.27 \\
\hline Epinephrine, yes/no & $97(57.4)$ & $39(52.7)$ & $10(71.4)$ & 0.42 \\
\hline Epinephrine, $\mu \mathrm{g} / \mathrm{kg} / \mathrm{min}^{\mathrm{b}}$ & $0.10[0,0.31]$ & $0.07[0,0.22]$ & $0.15[0.02,0.45]$ & 0.17 \\
\hline Dobutamine, yes/no & $50(29.6)$ & $31(41.9)$ & $5(35.7)$ & 0.17 \\
\hline Dobutamine, $\mu \mathrm{g} / \mathrm{kg} / \mathrm{min}^{\mathrm{b}}$ & $0[0,5]$ & $0[0,7]$ & $0[0,9.5]$ & 0.32 \\
\hline Norepinephrine, yes/no & $87(51.5)$ & $33(44.6)$ & $7(50)$ & 0.61 \\
\hline Norpinephrine, $\mu \mathrm{g} / \mathrm{kg} / \mathrm{min}^{\mathrm{b}}$ & $0.02[0,0.19]$ & $0[0,0.19]$ & $0.18[0,0.45]$ & 0.36 \\
\hline \multicolumn{5}{|l|}{ VA-ECMO implantation } \\
\hline Primary graft dysfunction & $12(7.1)$ & $16(21.6)$ & $2(14.3)$ & 0.006 \\
\hline Lactate, $\mathrm{mmol} / \mathrm{L}^{\mathrm{b}}$ & $6.9(3.8)$ & $5.1(3.2)$ & $5.2(2.7)$ & 0.002 \\
\hline Intraoperative implantation & $90(53.2)$ & $57(77.0)$ & $12(85.7)$ & 0.0004 \\
\hline Postoperative implantation & $79(46.7)$ & $17(22.9)$ & $2(14.3)$ & 0.0004 \\
\hline Epinephrine, yes/no & $123(72.8)$ & $42(56.8)$ & $10(71.4)$ & 0.049 \\
\hline Dobutamine, yes/no & $36(21.3)$ & 27 (36.5) & $4(28.6)$ & 0.046 \\
\hline
\end{tabular}


Norepinephrine, yes/no

77 (45.6)

$28(37.4)$

$5(35.7)$

0.46

D1 VA-ECMO support

Creatinine, $\mu \mathrm{mol} / \mathrm{L}^{\mathrm{b}}$

$\begin{array}{cccc}154.5 \pm 77.5 & 113.3 \pm 45.9 & 137.1 \pm 42.4 & 0.0001 \\ 47.2 \pm 8.6 & 51.4 \pm 9.2 & 50.9 \pm 14.0 & 0.008 \\ 32.2 \pm 21.3 & 42.6 \pm 44.6 & 21.4 \pm 29.0 & 0.01 \\ 18.7(4.5) & 20.4(3.9) & 19.2(4.7) & 0.04 \\ 5.2(3.7) & 3.85(2.5) & 3.91(2.5) & 0.04 \\ 600[135-1150] & 1550[1150-2250] & 1325[525-2300] & <0.0001\end{array}$

Results are count (\%), mean \pm SD or median [interquartile range 25,75 ].

${ }^{\text {a }}$ Chronic kidney failure is defined by a Glomerular Filtration Rate $<60 \mathrm{ml} / \mathrm{min}$

${ }^{\mathrm{b}}$ Refers to the maximum dose or maximal value

AKI, Acute kidney injury; BMI, body mass index; COPD, chronic obstructive pulmonary disease; D10, Day ten of follow-up after VA-ECMO support; LVEF, left ventricular ejection fraction; SAPS 2, simplified acute physiology score 2; SOFA, Sepsis-related Organ Failure Assessment; VA-ECMO, Veno-Arterial ExtraCorporeal Membrane Oxygenation. 

Table 3: Risk factors for D30 mortality

\begin{tabular}{|c|c|c|c|c|c|}
\hline \multirow[b]{2}{*}{ Factors } & \multirow{2}{*}{$\begin{array}{c}\text { Deceased } \\
\text { at D30 } \\
\text { No.=124 }\end{array}$} & \multirow{2}{*}{$\begin{array}{c}\text { Alive } \\
\text { at D30 } \\
\text { No.=133 }\end{array}$} & \multirow{2}{*}{$\begin{array}{c}\text { Univariable } \\
\text { analysis } \\
\text { P-value }\end{array}$} & \multicolumn{2}{|c|}{$\begin{array}{c}\text { Multivariable } \\
\text { analysis }\end{array}$} \\
\hline & & & & $\begin{array}{c}\text { Odds Ratio } \\
\text { [95\%CI] }\end{array}$ & P-value \\
\hline \multicolumn{6}{|l|}{ Demographic characteristics } \\
\hline Age, years & $66.8 \pm 11.8$ & $60.3 \pm 13.5$ & $<0.001$ & $\begin{array}{c}1.007[1.002, \\
1.012]\end{array}$ & 0.003 \\
\hline BMI, $\mathrm{kg} / \mathrm{m}^{2}$ & $26.8 \pm 5.7$ & $24.5 \pm 4.3$ & $<0.001$ & $\begin{array}{c}1.015[1.003, \\
1.026]\end{array}$ & 0.013 \\
\hline SAPS 2 & $63.7 \pm 18.4$ & $48.4 \pm 16.3$ & $<0.001$ & - & - \\
\hline SOFA & $16.5 \pm 3.7$ & $13.6 \pm 3.5$ & $<0.001$ & - & - \\
\hline LVEF, \% & $40[25,50]$ & $25[20,45]$ & $<0.001$ & - & - \\
\hline Diabetes & 40 (32.3\%) & $28(21.1 \%)$ & 0.058 & & \\
\hline Dyslipidemia & $77(62.1 \%)$ & $60(45.1 \%)$ & 0.009 & - & - \\
\hline High blood pressure & $81(65.3 \%)$ & 59 (44.4\%) & 0.001 & - & - \\
\hline Chronic arteriopathy & $24(19.4 \%)$ & $9(6.8 \%)$ & 0.005 & $1.20[1.01-1.44]$ & 0.041 \\
\hline \multicolumn{6}{|l|}{ Intraoperative period } \\
\hline $\begin{array}{l}\text { Red Blood Cell } \\
\text { transfusion, number }\end{array}$ & $3[0,5]$ & $2[0,4]$ & 0.091 & - & - \\
\hline $\begin{array}{l}\text { Cell salvage autologous } \\
\text { transfusion, mL }\end{array}$ & $475[247,725]$ & $392[228,663]$ & 0.082 & - & - \\
\hline \multicolumn{6}{|l|}{ VA-ECMO implantation } \\
\hline Biventricular failure & $53(42.7 \%)$ & $35(26.3 \%)$ & 0.008 & $1.15[1.02-1.30]$ & 0.028 \\
\hline Primary graft dysfunction & $22(16.5 \%)$ & 8 (6.5\%) & 0.020 & - & - \\
\hline Lactate, $\mathrm{mmol} / \mathrm{L}$ & $6.1[3.4,9.2]$ & $5.3[3.0,8.4]$ & 0.069 & - & - \\
\hline Inhaled Nitric Oxid & $10(8.1 \%)$ & $24(18.0 \%)$ & 0.030 & - & - \\
\hline AKI at implantation & $76(61.3 \%)$ & $63(47.4 \%)$ & 0.035 & - & - \\
\hline Urine output & $750[200,1390]$ & $1125[500,1860]$ & 0.008 & - & - \\
\hline Type of surgery & & & 0.003 & - & - \\
\hline Heart transplantation & 15 (12.1\%) & $44(33.1 \%)$ & - & - & - \\
\hline Valvular surgery & $38(30.6 \%)$ & $32(24.1 \%)$ & - & - & - \\
\hline Coronary bypass & $27(21.8 \%)$ & $22(16.5 \%)$ & - & - & - \\
\hline Combined surgery & $13(10.5 \%)$ & $9(6.8 \%)$ & - & - & - \\
\hline Other surgery & $31(25.0 \%)$ & 26 (19.5\%) & - & - & - \\
\hline
\end{tabular}


Results are count (\%), mean \pm SD or median [interquartile range 25, 75]. Adjusted Odds Ratio of factors that did not achieve statistical significance in multivariable analysis are not presented.

Due to missing data, SOFA and SAPS2 were not included in the multivariable analysis.

AKI, Acute Kidney Injury; BMI, body mass index; COPD, chronic obstructive pulmonary disease; LVEF, left ventricular ejection fraction; SAPS 2, simplified acute physiology score 2; SOFA, Sepsis-related Organ Failure Assessment; VA-ECMO, Veno-Arterial ExtraCorporeal Membrane Oxygenation. 